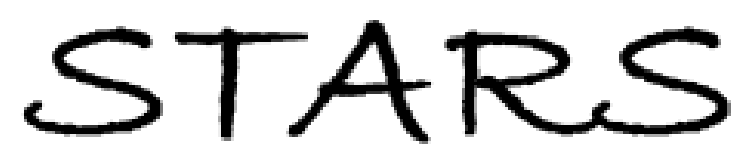

University of Central Florida

STARS

$1-1-2014$

\title{
A low voltage submillisecond-response polymer network liquid crystal spatial light modulator
}

Jie Sun

University of Central Florida

Shin-Tson Wu

University of Central Florida

Yasuhiro Haseba

Find similar works at: https://stars.library.ucf.edu/facultybib2010 University of Central Florida Libraries http://library.ucf.edu

This Article is brought to you for free and open access by the Faculty Bibliography at STARS. It has been accepted for inclusion in Faculty Bibliography 2010 s by an authorized administrator of STARS. For more information, please contact STARS@ucf.edu.

\section{Recommended Citation}

Sun, Jie; Wu, Shin-Tson; and Haseba, Yasuhiro, "A low voltage submillisecond-response polymer network liquid crystal spatial light modulator" (2014). Faculty Bibliography 2010s. 6149.

https://stars.library.ucf.edu/facultybib2010/6149

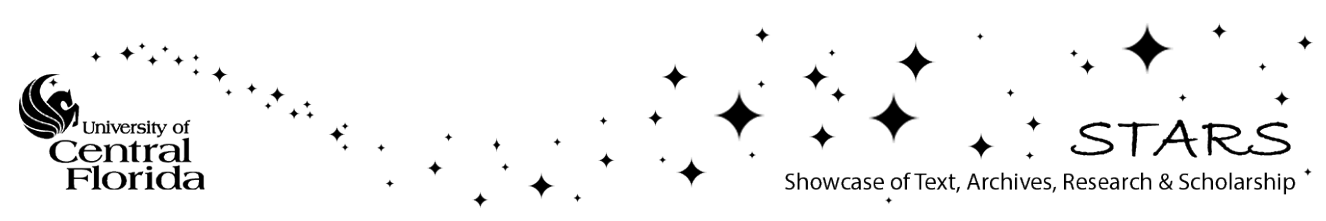




\section{A low voltage submillisecond-response polymer network liquid crystal spatial light modulator}

Cite as: Appl. Phys. Lett. 104, 023305 (2014); https://doi.org/10.1063/1.4862474

Submitted: 06 December 2013 . Accepted: 05 January 2014 . Published Online: 17 January 2014

Jie Sun, Shin-Tson Wu, and Yasuhiro Haseba
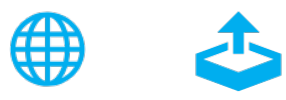

View Online

Export Citation

\section{ARTICLES YOU MAY BE INTERESTED IN}

Fast-response and scattering-free polymer network liquid crystals for infrared light modulators

Applied Physics Letters 84, 1233 (2004); https://doi.org/10.1063/1.1649816

Submillisecond-response polymer network liquid crystal phase modulators at $1.06-\mu \mathrm{m}$ wavelength

Applied Physics Letters 99, 021106 (2011); https://doi.org/10.1063/1.3611031

Reconfigurable fabrication of scattering-free polymer network liquid crystal prism/grating/ lens

Applied Physics Letters 102, 161106 (2013); https://doi.org/10.1063/1.4802919

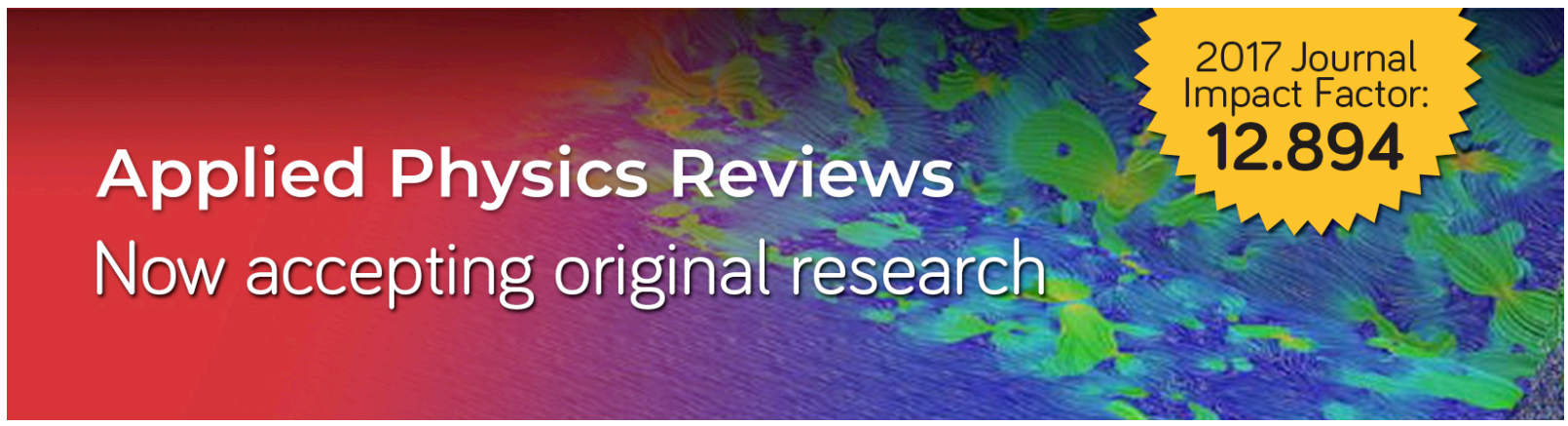




\title{
A low voltage submillisecond-response polymer network liquid crystal spatial light modulator
}

\author{
Jie Sun, ${ }^{1}$ Shin-Tson Wu, ${ }^{1, a)}$ and Yasuhiro Haseba ${ }^{2}$ \\ ${ }^{1}$ College of Optics and Photonics, University of Central Florida, Orlando, Florida 32816, USA \\ ${ }^{2}$ JNC Petrochemical Corporation, Ichihara Research Center, Ichihara, Chiba 290-8551, Japan
}

(Received 6 December 2013; accepted 5 January 2014; published online 17 January 2014)

\begin{abstract}
We report a low voltage and highly transparent polymer network liquid crystal (PNLC) with submillisecond response time. By employing a large dielectric anisotropy LC host JC-BP07N, we have lowered the $\mathrm{V}_{2 \pi}$ voltage to $23 \mathrm{~V}$ at $\lambda=514 \mathrm{~nm}$. This will enable PNLC to be integrated with a high resolution liquid-crystal-on-silicon spatial light modulator, in which the maximum voltage is $24 \mathrm{~V}$. A simple model correlating PNLC performance with its host LC is proposed and validated experimentally. By optimizing the domain size, we can achieve $\mathrm{V}_{2 \pi}<15 \mathrm{~V}$ with some compromises in scattering and response time. (C) 2014 AIP Publishing LLC.

[http://dx.doi.org/10.1063/1.4862474]
\end{abstract}

Liquid crystal spatial light modulators (LC SLMs) ${ }^{1}$ have been widely used in digital hologram generation, ${ }^{2,3}$ adaptive optics, ${ }^{4,5}$ adaptive lens, ${ }^{6}$ and laser beam steering. ${ }^{7-9}$ For these applications, fast response time, $2 \pi$ phase change, and low operation voltage are some basic requirements. Various LC technologies have been explored, such as ferroelectric LC, ${ }^{10}$ dual frequency liquid crystal (DFLC), ${ }^{11}$ stressed LC, ${ }^{12,13}$ and polymer network liquid crystal (PNLC). ${ }^{14,15}$ Each approach has its own pros and cons. Ferroelectric LC shows microsecond response time, but it is a bistable device and is difficult to obtain continuous phase-only modulation. DFLC offers fast rise time and decay time, but its crossover frequency is quite sensitive to the temperature. Stressed LC does not require an alignment layer, but it needs a delicate mechanical shearing process. Scattering-free PNLCs have been developed for $\lambda=1.55 \mu \mathrm{m}$ and $1.06 \mu \mathrm{m} ;{ }^{14,16}$ however, these PNLCs scatter light strongly in the visible region because of voltage-induced micron-sized multi-domain structures. ${ }^{14,16-19}$ To suppress scattering in the visible region, the domain size should be reduced to $\sim 100 \mathrm{~nm} .^{15,20}$ The major challenge is that the operation voltage increases dramatically due to strong anchoring from these fine polymer network structures. Such a PNLC requires $V_{2 \pi} \sim 50 V_{\text {rms }}$ to obtain $2 \pi$ phase change in reflective mode at $\lambda=514 \mathrm{~nm},{ }^{15}$ while the commonly used high resolution liquid-crystal-onsilicon (LCoS) has a maximum voltage of $24 \mathrm{~V} .^{21}$ To integrate the submillisecond-response PNLC with LCoS for widespread applications, we need to reduce $V_{2 \pi}$ to below $24 \mathrm{~V}$ while keeping scattering loss negligible.

In this paper, we demonstrate a reflective mode PNLC phase modulator with $\mathrm{V}_{2 \pi} \approx 23 \mathrm{~V}$ at $\lambda=514 \mathrm{~nm}$. To achieve such a low operation voltage, we developed and employed a high dielectric anisotropy $(\Delta \varepsilon \approx 302)$ nematic LC host, JC-BP07N (by JNC). The PNLC sample is highly transparent and its scattering loss is negligible in the $\lambda \geq 500 \mathrm{~nm}$ range. The measured decay time is $250 \mu$ s and rise time is $680 \mu \mathrm{s}$ at room temperature $\left(\approx 22{ }^{\circ} \mathrm{C}\right)$, and hysteresis is about $1.7 \%$. To understand the corresponding physical mechanisms and find

a)E-mail: swu@ucf.edu the right direction for device optimization, we propose a semi-empirical model to correlate the electro-optical performance of PNLC with the host LC parameters. Based on this model, a reflective mode PNLC with $\mathrm{V}_{2 \pi} \leq 15 \mathrm{~V}$ can be achieved if we can tolerate a slightly higher scattering loss $(\approx 3 \%)$ and slower response time $(\approx 580 \mu \mathrm{s})$.

First, let us discuss our approach for reducing the operation voltage. For a homogeneous cell filled with a positive $\Delta \varepsilon$ nematic LC, the LC directors start to reorient when the applied voltage exceeds the Freedericksz threshold voltage ${ }^{22}$

$$
V_{t h}=\pi \sqrt{K_{11} /\left(\varepsilon_{0} \Delta \varepsilon\right)},
$$

where $\mathrm{K}_{11}$ is the splay elastic constant. For phase modulation with a given LC birefringence and device configuration, $\mathrm{V}_{2 \pi}$ is proportional to $\mathrm{V}_{\mathrm{th}} \cdot{ }^{23}$ One straightforward way to reduce $\mathrm{V}_{2 \pi}$ and $\mathrm{V}_{\text {th }}$ is to employ a large $\Delta \varepsilon$ LC host. An inevitable tradeoff is the increased viscosity.

We developed a nematic LC mixture JC-BP07N with a very large $\Delta \varepsilon$. The physical properties of $\mathrm{JC}-\mathrm{BP} 07 \mathrm{~N}$ are listed as follows: clearing temperature $\mathrm{T}_{\mathrm{c}}=87^{\circ} \mathrm{C}$, birefringence $\Delta \mathrm{n}=0.17$ at $\lambda=514 \mathrm{~nm}$, and visco-elastic coefficient $\gamma_{1} / \mathrm{K}_{11}=224 \mathrm{~ms} / \mu \mathrm{m}^{2}\left(22^{\circ} \mathrm{C}\right)$. To characterize its dielectric constants, we filled JC-BP07N into two LC cells: one with homogeneous and another with homeotropic alignment. By comparing the capacitance before and after filling the cells, we obtained both parallel $\left(\varepsilon_{/ /}\right)$and perpendicular $\left(\varepsilon_{\perp}\right)$ dielectric constants of this LC. The probing voltage was $0.1 \mathrm{~V}_{\mathrm{rms}}$, which is below $\mathrm{V}_{\text {th }}\left(0.2 \mathrm{~V}_{\mathrm{rms}}\right)$. Dielectric anisotropy is defined as $\Delta \varepsilon=\varepsilon_{/ /}-\varepsilon_{\perp}$.

Figure 1(a) shows the frequency dependent dielectric constant of JC-BP07N. Since $\varepsilon_{\perp}(\approx 14)$ is almost independent of frequency, only $\varepsilon_{/ /}$results are shown in Fig. 1(a). At $f=100 \mathrm{~Hz}$ and $22^{\circ} \mathrm{C}$, JC-BP07N has a huge parallel dielectric constant $\varepsilon_{/ /} \approx 316$. Thus, $\Delta \varepsilon \approx 302$. According to Debye equation, the dielectric constant of a LC can be described as follows: ${ }^{24}$

$$
\varepsilon_{/ /}^{*}(f)=\varepsilon_{/ /}(\infty)+\frac{\varepsilon_{/ /}(0)-\varepsilon_{/ /}(\infty)}{1+i\left(f / f_{r}\right)},
$$



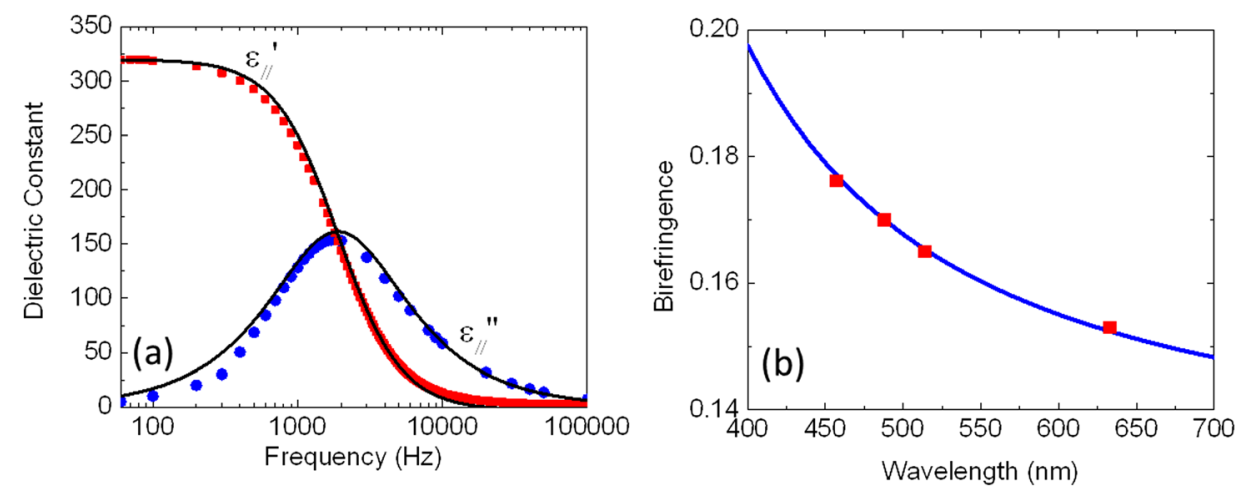

FIG. 1. (a) Frequency-dependent complex $\varepsilon_{/ /}$of JC-BP07N: dots are measured results and solid line is fitting with Eq. (2); (b) wavelength dependent $\Delta \mathrm{n}$ of JC-BP07N: dots are measured results and solid line is fitting with Eq. (3). where $\varepsilon_{/ /}^{*}=\varepsilon_{/ /}^{\prime}-i \varepsilon / /{ }^{\prime \prime}$ is the complex dielectric constant; $f$ is the driving frequency; $\varepsilon_{/ /}(0)$ and $\varepsilon_{/ /}(\infty)$ represent the real part of parallel dielectric constant at low and high frequency limits; and $f_{r}$ is the relaxation frequency. In Fig. 1(a), dots represent the measured data and solid lines fitting curves. Good fitting is found for both real and imaginary parts. From the fittings, we find $f_{r}=1.9 \mathrm{kHz}$. This relatively low relaxation frequency originates from the high viscosity of JC-BP07N; high viscosity makes the LC molecules difficult to follow the electric field as frequency increases.

Figure 1(b) shows the wavelength dependent birefringence of JC-BP07N. Dots represent the measured data, while solid line is the fitting with the birefringence dispersion equation $^{25}$

$$
\Delta n(\lambda)=G \lambda^{2} \lambda^{* 2} /\left(\lambda^{2}-\lambda^{* 2}\right)
$$

where $\mathrm{G}$ is a proportionality constant and $\lambda^{*}$ is the mean resonance wavelength.

To fabricate PNLCs, we prepared a precursor by mixing 92.3 wt. \% LC host (JC-BP07N, JNC) with 7.2\% monomer (Merck RM257) and $0.5 \%$ photo-initiator (Genocure BAPO). This recipe has been optimized to achieve scattering-free samples for visible wavelengths. The precursor was filled into a homogeneous LC cell (indium tin oxide glass substrates) with $5-\mu$ m cell gap and $3^{\circ}$ pretilt angle. A UV light-emitting diode (LED) lamp ( $\lambda=385 \mathrm{~nm}$ ) was used for curing the precursor and the curing temperature was controlled at $10^{\circ} \mathrm{C}$. We called this sample as PNLC-1. The reason we used low temperature curing is to decrease the polymer diffusion rate, which plays a crucial role to shrink the LC domain sizes. ${ }^{19}$

After UV curing, PNLC-1 maintained a good homogeneous alignment following the substrate rubbing direction.
To prove this, we sandwiched the PNLC-1 cell between two crossed polarizers on a light table. When the alignment direction of PNLC-1 was oriented parallel to the optic axis of the polarizer, a very good dark state was observed.

We characterized the power-on scattering loss of PNLC1 through transmission spectra measurement. A photodiode detector with an aperture of $0.9 \mathrm{~cm}$ was set at $20 \mathrm{~cm}$ behind the sample. Such a small collection angle $\left(\sim 2.6^{\circ}\right)$ is to ensure that the light scattered off the LC domains is not collected by the detector. As shown in Fig. 2(a), the transmission spectrum of PNLC-1 with a biased voltage leading to maximum scattering loss $\left(\mathrm{V}_{\text {max.scatt. }}\right)$ is comparable to that of the LC host cell, indicating the scattering-free feature of this PNLC. To more qualitatively analyze the scattering loss, we normalized the transmission spectrum of PNLC-1 at

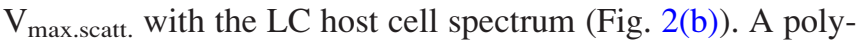
nomial fitting of the LC host spectrum (purple dashed lines in Fig. 2(a)) was used in the normalization process in order to minimize the oscillation caused by Fabry-Perot effect. Results indicate that the scattering loss of PNLC-1 is below $1 \%$ when the wavelength is longer than $500 \mathrm{~nm}$. Blue dotted lines represent the calculated results according to Rayleigh scattering model ${ }^{19}$

$$
T=\frac{1}{2} \exp \left(-C_{e} \frac{\Delta n^{2}}{\lambda_{0}^{4}} d\right)+\frac{1}{2} \exp \left(-C_{o} \frac{\Delta n^{2}}{\lambda_{0}^{4}} d\right)
$$

where $\mathrm{C}_{\mathrm{e}}$ and $\mathrm{C}_{\mathrm{o}}$ represent the domain size parameters for the extraordinary ray (e-ray) and ordinary ray (o-ray), respectively, $\lambda_{0}$ is the wavelength in vacuum, and $d$ is the LC thickness.

Figure 3(a) shows the measured voltage-dependent reflectance (VR) of PNLC-1 at $\lambda=514 \mathrm{~nm}$. To animate
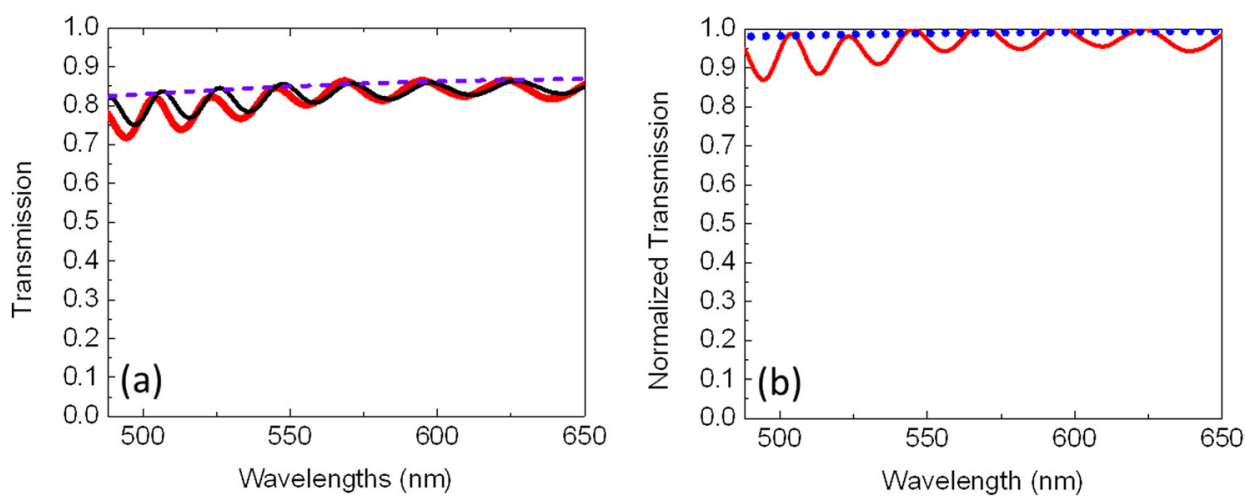

FIG. 2. (a) Transmission spectra of PNLC-1 (red thick line) with a biased voltage leading to maximum scattering; the spectrum of the JC-BP07N LC host cell (black thin line) is included for comparison purpose; purple dashed lines represent the polynomial fitting for the LC host. (b) Normalized transmission spectrum of the on-state PNLC-1; the purple dashed lines in Fig. 2(a) are used for normalization; red solid line represents experimental results and blue dotted lines represent fitting with Eq. (4). 


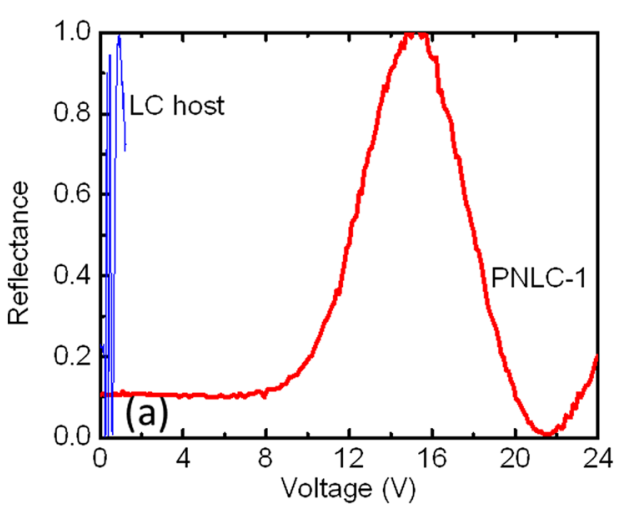

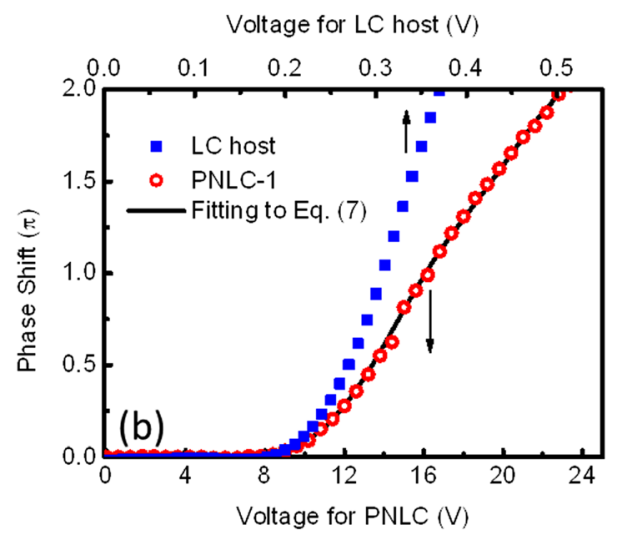

FIG. 3. (a) Voltage dependent reflectance curves of PNLC-1 at $f=100 \mathrm{~Hz}$ (thick red line) and LC host JC-BP07N (thin blue line). (b) The VP curves of PNLC-1 (red circles) and LC host (blue squares) in reflective mode; solid black line is the fitting with Eq. (7). reflective mode operation, we placed a mirror behind the PNLC-1 cell to fold the optical path. The incident beam and reflected beam formed a very small angle $\left(\sim 4^{\circ}\right)$. The rubbing direction of the PNLC-1 cell was oriented at $45^{\circ}$ with respect to the optical axis of the polarizer and the analyzer was crossed. For comparison purpose, the VR curve of a JC$\mathrm{BP} 07 \mathrm{~N}$ host LC cell with the same configuration is also included. The peak reflectance of PNLC-1 cell is comparable to that of the LC host cell, indicating that the scattering of PNLC-1 is indeed negligible. From Fig. 3(a), we obtain $\mathrm{V}_{2 \pi}$ $\approx 23 \mathrm{~V}_{\mathrm{rms}}$ for PNLC-1 at $\lambda=514 \mathrm{~nm}$. Figure $3(\mathrm{~b})$ shows the voltage-dependent phase change (VP) curves for LC host (JC-BP07N) and PNLC-1. We will discuss these results in detail later. Note that these VP curves are for e-ray whose polarization is parallel to the LC cell rubbing direction.

Next, we measured the hysteresis and response time of PNLC-1. For a polymer-stabilized LC, hysteresis originates from electric field-induced network distortion. Details depend on the rigidity of polymer networks and peak electric field. In our PNLC-1, its $\mathrm{V}_{2 \pi} \approx 23 \mathrm{~V}$ and cell gap is $5 \mu \mathrm{m}$, so the corresponding electric field is only $4.6 \mathrm{~V} / \mu \mathrm{m}$. As a result, the measured hysteresis is only $1.7 \%$. The response time is defined as $100 \%$ to $10 \%$ phase change. We measured the $2 \pi$ phase change of PNLC-1 and results are: rise time $\sim 680 \mu \mathrm{s}$ and decay time $\sim 250 \mu$ s at room temperature. The relaxation time of PNLC can be approximated as ${ }^{22}$

$$
\tau \approx \frac{\gamma_{1}}{K_{11}} \frac{d_{1}^{2}}{\pi^{2}}
$$

where $d_{1}$ is the average LC domain size, which is determined by the polymer concentration and UV curing conditions. From Eq. (5), we can estimate $d_{1}$ from the measured decay time. Knowing that $\tau \approx 250 \mu \mathrm{s}$ and $\gamma_{1} / \mathrm{K}_{11}=224 \mathrm{~ms} / \mu \mathrm{m}^{2}$, we find $\mathrm{d}_{1} \approx 105 \mathrm{~nm}$. This result is in good agreement with another method described below. The $\gamma_{1} / \mathrm{K}_{11}$ of JC-BP07N is about $10 \times$ larger than that of E7 mixture (Merck). According to our previous study, a high viscosity LC host tends to slow down the polymer diffusion rate and forms finer polymer networks. ${ }^{19}$ The small domain size outweighs the high viscosity. As a result, such a highly viscous LC host JC-BP07N can still lead to a submillisecond-response PNLC with only $\sim 7 \%$ polymer. The rise time is more complicated to model because it involves an additional parameter: the ratio of grayscale voltage over threshold voltage. ${ }^{22}$ Therefore, we only used relaxation time to estimate the average domain size.
In order to optimize PNLC performance, here we propose a multi-layered model to correlate its electro-optical properties with the LC host. For a PNLC with average domain size $d_{1}$, we can consider it as dividing a LC cell into $\mathrm{N}=\mathrm{d} / \mathrm{d}_{1}$ layers, where $d$ is the cell gap. In so doing, the PNLC should have $\mathrm{N}$ times higher threshold voltage than its LC host. For a given LC precursor and cell parameter, we can write the operation voltage as

$$
V_{o n}=\frac{d}{d_{1}} V_{o n, 0} \propto \pi \frac{d}{d_{1}} \sqrt{\frac{K_{11}}{\varepsilon_{0} \Delta \varepsilon}},
$$

where $\mathrm{V}_{\text {on, } 0}$ is the operation voltage of the LC host. Through the fitting of threshold voltage, we obtain $\mathrm{d}_{1} \sim 103 \mathrm{~nm}$ for PNLC-1. Note that this value is almost identical to the one ( $\sim 105 \mathrm{~nm})$ we estimated from the response time data, confirming the validity of this model. However, due to the strong anchoring effect of the 7\% RM257 polymer and the greatly increased LC/polymer surface area, the electric field-induced refractive index change $\left(\delta \mathrm{n}_{1}\right)$ for PNLC should be much smaller than that of the LC host $\left(\delta \mathrm{n}_{0}\right)$. For simplicity, we assume $\delta \mathrm{n}_{1}(\mathrm{~V})$ is linearly proportional to $\delta \mathrm{n}_{0}$, i.e., $\delta \mathrm{n}_{1}=\mathrm{A} \delta \mathrm{n}_{0}$. Then, the voltage-dependent phase change of PNLC can be written as

$$
\phi\left(\lambda, V_{\text {on }}\right)=A \frac{2 \pi d}{\lambda} \delta n_{0}\left(\lambda, V_{\text {on }, 0}\right)
$$

Combining Eqs. (6) and (7), we can fit the VP curve of PNLC-1 well using the VP information of the LC host cell (shown in Fig. 3(b)). The only fitting parameter $\mathrm{A}$ is extracted to be 0.51 .

To validate the effectiveness of this model, we measured the VP curves at three laser wavelengths $(633 \mathrm{~nm}, 514 \mathrm{~nm}$, and $488 \mathrm{~nm}$ ) and compared the experimental data with simulation results (Fig. 4(a)). The wavelength dependent $\Delta \mathrm{n}$ shown in Fig. 1(b) has been taken into consideration in the model. Good agreement is found between experiment and simulation within the voltage range we care about. As the wavelength increases, phase dynamic range decreases due to the smaller birefringence.

This simple model can also predict the frequency effect on VP curves as shown in Fig. 4(b). When the driving frequency increases from $100 \mathrm{~Hz}$ to $1000 \mathrm{~Hz}$, the dynamic range (at $24 \mathrm{~V}$ ) gradually decreases because of the reduced $\Delta \varepsilon$ as Fig. 1(a) shows. 

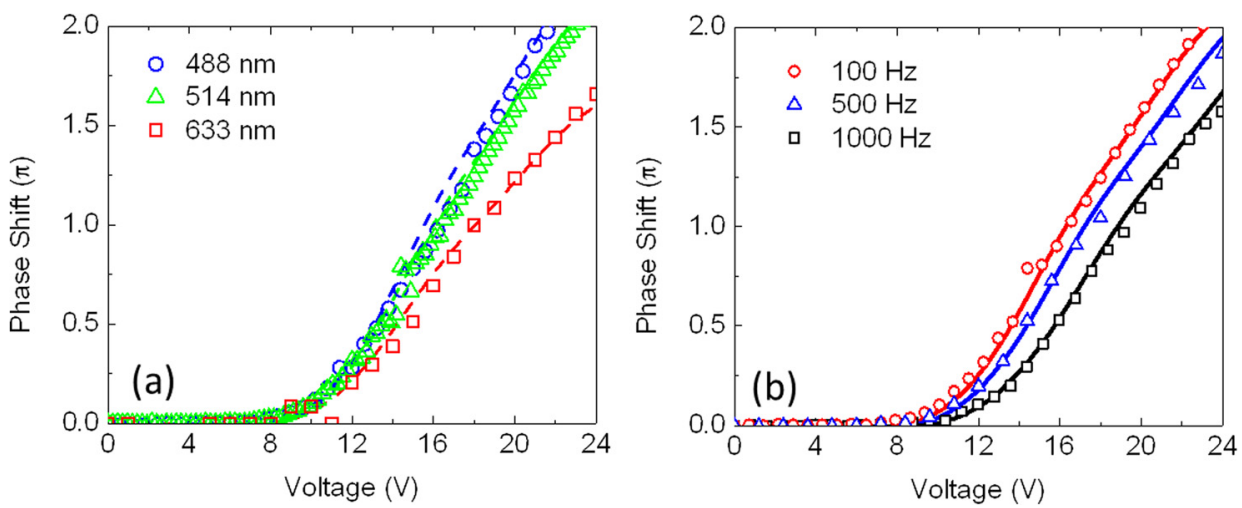

FIG. 4. (a) Wavelength effect on the VP curves of PNLC-1 at $f=100 \mathrm{~Hz}$. (b) Frequency effect on the VP curves of PNLC-1 at $\lambda=514 \mathrm{~nm}$. Dots are measured data and lines are simulation results.
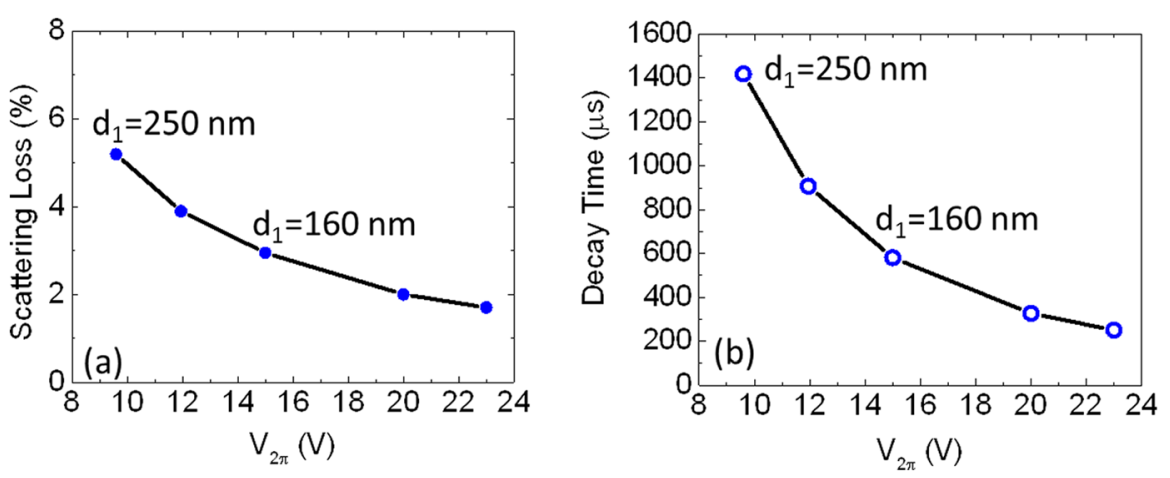

FIG. 5. (a) $V_{2 \pi}$ versus scattering loss for PNLCs in reflective mode; (b) $V_{2 \pi}$ versus relaxation time for 5 domain sizes: $250 \mathrm{~nm}, 200 \mathrm{~nm}, 160 \mathrm{~nm}$, $120 \mathrm{~nm}$, and $103 \mathrm{~nm}$.

Although $\mathrm{V}_{2 \pi} \approx 23 \mathrm{~V}$ would allow PNLC to be integrated in a high resolution LCoS panel, a lower operation voltage helps to reduce power consumption. According to Eq. (6), for a given LC host, increasing the average LC domain size $d_{1}$ seems to be the most effective approach to reduce operation voltage. However, the tradeoffs in increased scattering loss and response time should be balanced. Here, we simulated the performance of PNLCs with different domain sizes based on JC-BP07N host. Figures 5(a) and 5(b) depict the relationship between $V_{2 \pi}$ and scattering loss/relaxation time at $\lambda=514 \mathrm{~nm}$ by changing the domain size. In reflective mode, the optical path is doubled so that the scattering loss is increased. This has been considered in our simulation. If we increase $\mathrm{d}_{1}$ from $103 \mathrm{~nm}$ to $160 \mathrm{~nm}, \mathrm{~V}_{2 \pi}$ can be reduced from $23 \mathrm{~V}$ to $15 \mathrm{~V}$ but the scattering loss is increased to $3 \%$ at $\lambda=514 \mathrm{~nm}$ and decay time to $580 \mu \mathrm{s}$. Further increasing domain size to $250 \mathrm{~nm}$ helps to reduce $\mathrm{V}_{2 \pi}$ to $9.6 \mathrm{~V}$, but the scattering loss increases to $5.3 \%$ and response time jumps to $1.4 \mathrm{~ms}$.

To further increase the phase dynamic range of PNLC, a thicker cell gap can be considered. Unlike a nematic LC whose response time is strongly cell gap dependent, increasing the cell gap of a PNLC will not sacrifice its response time, which is mainly governed by the domain sizes. However, a tradeoff is the increased operation voltage, as Eq. (6) indicates.

In conclusion, we have demonstrated a low voltage and highly transparent PNLC with submillisecond response time and negligible hysteresis. By employing a large $\Delta \varepsilon \mathrm{LC}$ host JC-BP07N, we have reduced the $\mathrm{V}_{2 \pi}$ of PNLC- 1 to $23 \mathrm{~V}$ in reflective mode operation at $\lambda=514 \mathrm{~nm}$. Such a low voltage will allow PNLC-1 to be integrated in a high resolution LCoS for next-generation SLM applications. We have also proposed a semi-empirical multi-layered model to simulate the performances of PNLCs. Good agreement with experimental data is obtained. This model provides useful guidelines for optimizing the PNLC performance.

The authors are indebted to the U.S. Air Force Office of Scientific Research (AFOSR) for financial support under Contract No. FA95550-09-1-0170.

${ }^{1}$ U. Efron, Spatial Light Modulator Technology: Materials, Devices, and Applications (Marcel Dekker, 1995).

${ }^{2}$ O. Matoba, T. J. Naughton, Y. Frauel, N. Bertaux, and B. Javidi, Appl. Opt. 41, 6187 (2002).

${ }^{3}$ P. Clemente, V. Durán, E. Tajahuerce, P. Andrés, V. Climent, and J. Lancis, Opt. Lett. 38, 2524 (2013).

${ }^{4}$ G. D. Love, Appl. Opt. 36, 1517 (1997).

${ }^{5}$ S. Quirin, D. S. Peterka, and R. Yuste, Opt. Express 21, 16007 (2013).

${ }^{6} \mathrm{H}$. Ren and S. T. Wu, Introduction to Adaptive Lenses (Wiley, 2012).

${ }^{7}$ P. F. McManamon, T. A. Dorschner, D. L. Corkum, L. J. Friedman, D. S. Hobbs, M. Holz, S. Liberman, H. Q. Nguyen, D. P. Resler, R. C. Sharp, and E. A. Watson, Proc. IEEE 84, 268 (1996).

${ }^{8}$ S. Serati and J. Stockley, Proc. SPIE 5894, 58940K (2005).

${ }^{9}$ F. Feng, I. H. White, and T. D. Wilkinson, J. Lightwave Technol. 31, 2001 (2013).

${ }^{10}$ J. Fünfschilling and M. Schadt, Jpn. J. Appl. Phys., Part 1 30, 741 (1991).

${ }^{11}$ W. J. De Jeu, C. J. Gerritsma, P. Van Zanten, and W. J. A. Goossens, Phys. Lett. A 39, 355 (1972).

${ }^{12}$ J. L. West, G. Zhang, A. Glushchenko, and Y. Reznikov, Appl. Phys. Lett. 86, 031111 (2005).

${ }^{13}$ Y.-H. Wu, Y.-H. Lin, H. Ren, X. Nie, J.-H. Lee, and S.-T. Wu, Opt. Express 13, 4638 (2005).

${ }^{14}$ Y.-H. Fan, Y.-H. Lin, H. Ren, S. Gauza, and S.-T. Wu, Appl. Phys. Lett. 84, 1233 (2004).

${ }^{15}$ J. Sun and S.-T. Wu, J. Polym. Sci., Part B: Polym. Phys. 52, 183 (2014).

${ }^{16}$ J. Sun, H. Xianyu, Y. Chen, and S.-T. Wu, Appl. Phys. Lett. 99, 021106 (2011).

${ }^{17}$ R. A. M. Hikmet and H. M. J. Boots, Phys. Rev. E 51, 5824 (1995).

${ }^{18}$ I. Dierking, Adv. Mater. 12, 167 (2000). 
${ }^{19}$ J. Sun, Y. Chen, and S.-T. Wu, Opt. Express 20, 20124 (2012).

${ }^{20}$ J. Sun, S. Xu, H. Ren, and S.-T. Wu, Appl. Phys. Lett. 102, 161106 (2013).

${ }^{21}$ S. A. Serati, X. Xia, O. Mughal, and A. Linnenberger, Proc. SPIE 5106, 138 (2003).
${ }^{22}$ I. C. Khoo and S. T. Wu, Optics and Nonlinear Optics of Liquid Crystals (World Scientific, 1993).

${ }^{23}$ U. Efron, S. T. Wu, and T. D. Bates, J. Opt. Soc. Am. B 3, 247 (1986).

${ }^{24}$ F. C. Frank, Discuss. Faraday Soc. 25, 19 (1958).

${ }^{25}$ J. Li and S.-T. Wu, J. Appl. Phys. 95, 896 (2004). 